\title{
Peripheral tissue oximetry: comparing three commercial near- infrared spectroscopy oximeters on the forearm
}

\author{
Simon Hyttel-Sorensen • Trine Witzner Hessel • \\ Gorm Greisen
}

Received: 14 May 2013/Accepted: 23 August 2013/Published online: 30 August 2013

(c) The Author(s) 2013. This article is published with open access at Springerlink.com

\begin{abstract}
Estimation of regional tissue oxygenation $\left(\mathrm{rStO}_{2}\right)$ by near infrared spectroscopy enables non-invasive end-organ oxygen balance monitoring and could be a valuable tool in intensive care. However, the diverse absolute values and dynamics of different devices, and overall poor repeatability of measurements are a problem. The aim of the present study is to test the hypothesis that INVOS 5100C, FORE-SIGHT and NONIN EQUANOX 7600 have similar properties concerning absolute values, repeatability, and sensitivity to changes in $\mathrm{rStO}_{2}$. To test repeatability the sensors were repositioned 20 times during hemodynamic steady state on the adult forearm. Afterwards six vascular occlusions by inflation of an upper arm cuff were done to achieve low oxygenation in the forearm. Absolute values were compared by repeated-measures ANOVA, repeatability was estimated by the within-subject standard deviation, $\mathrm{S}_{\mathrm{w}}$, and response to changing oxygenation by the down slope of $\mathrm{rStO}_{2}$ during vascular occlusion in the respective arm. 10 healthy adults, 21-29 years old, with double skinfolds on the forearm less than $10 \mathrm{~mm}$ participated. The median $\mathrm{rStO}_{2}$ was $70.7 \%$ (interquartile range (IQR) $7.7 \%$ ), $68.4 \%$ (IQR $8.4 \%$ ), and $64.6 \%$ (IQR 4.8) with INVOS, NONIN, and FORE-SIGHT, respectively, the median rate of decline was $13.2 \% / \mathrm{min}$ (IQR 9.6), $22.8 \% / \mathrm{min}$ (IQR 18.0), and $10.8 \% / \mathrm{min}$ (IQR 6.0), and the same-site repeatability was $2.9 \%(95 \%$ CI 2.4-3.3), $4.6 \%$ (CI 3.9-5.3), and $2.0 \%$ (CI 1.7-2.3). INVOS gave significantly higher steady state values than FORE-SIGHT, and NONIN had the steepest decline in $\mathrm{rStO}_{2}$, but the poorest repeatability. Two measures of
\end{abstract}

S. Hyttel-Sorensen $(\bowtie) \cdot$ T. W. Hessel · G. Greisen Department of Neonatology, Rigshospitalet, University Hospital Copenhagen, Blegdamsvej 9, 2100 Copenhagen, Denmark e-mail: simonhyttelsrensen@me.com signal-to-noise were similar among devices. This suggests that good repeatability comes at the expense of low sensitivity to changes in oxygenation. Values of $\mathrm{rStO}_{2}$ on the forearm from INVOS, NONIN and FORE-SIGTH cannot be used interchangeably.

Keywords Near infrared spectroscopy (NIRS) · Oxygenation $\cdot$ Muscle $\cdot$ Medical device

\section{Introduction}

Near infrared spectroscopy (NIRS) enables estimation of the regional tissue haemoglobin oxygen saturation $\left(\mathrm{rStO}_{2}\right)$. $\mathrm{rStO}_{2}$ is a weighted average of the saturation in arterial and venous blood in the illuminated tissue. The arterial-tovenous ratio is about 25:75 and $\mathrm{rStO}_{2}$ is therefore closer correlated to the venous than to the arterial saturation [1]. Thus NIRS is a non-invasive, continuous estimate of the regional oxygen delivery-consumption balance and could potentially detect low regional blood flow that would otherwise go undetected [2]. However implementation into standard clinical care has yet to be seen. This is likely due to lack of convincing outcome data and randomized trials in combination with rather costly equipment. Furthermore previous studies have shown that a repeated measurement can vary up to 14 percentage points $(95 \% \mathrm{CI})$ [3-5]. This large intra-subject variability is an issue that needs improvement, before the potential of the technology can be fully realized.

Near infrared spectroscopy oximetry is based on the fact that hemoglobin exist in two main forms, oxyhemoglobin $(\mathrm{O} 2 \mathrm{Hb})$ and deoxyhemoglobin $(\mathrm{HHb})$, each having its own particular optical absorption characteristics. Most commercial devices apply continuous wave spectroscopy and 
use a multi-distance approach measuring the reflected light attenuation at several wavelengths as a function of distance from the light source. Assumptions regarding the wavelength dependence of scattering is necessary to derive the spectral shape of absorption coefficient and thereby deduce $\mathrm{rStO}_{2}$ [6]. These assumptions in combination with assumptions about the water content and the different light emitter and detector geometry probably account for the differences between devices.

It is well established that peripheral muscle $\mathrm{rStO}_{2}$ is a hemodynamic variable, that at least on group level differs between the healthy and the severely ill $[7,8]$, and is related to clinical relevant outcomes [7, 9, 10]. While these studies were done with different NIRS oximeters on different sites on the body the issue of generalizability is a hindrance to widespread clinical implementation. A recent review by Scheeren et al. [11] addresses some of the current strengths and limitations of the technology.

The new generation of NIRS sensors are FDA approved to claim reliable absolute values meaning that clinical validation studies have shown sufficient precision compared to a weighted average of arterial and jugular bulb saturation [12]. This should translate into better repeatability of measurements and suggests a significant step forward for the technology. Previous studies comparing different NIRS devices focus primarily on steady state comparison of mean values, whereas no estimates are given for repeatability or sensitivity to changing oxygenation. When comparing different devices all three parameters are of importance: the absolute $\mathrm{rStO}_{2}$ values during the normal state, the changes in $\mathrm{rStO}_{2}$ when oxygenation is altered, and the repeatability, i.e. the similarity of repeated measurements.

The aim of the present study is test the hypothesis that three commercial NIRS devices for peripheral measurements have similar absolute values of $\mathrm{rStO}_{2}$. Secondary outcomes include repeatability of measurements and slopes of changes in $\mathrm{rStO}_{2}$ during a vascular occlusion test. The devices compared are INVOS 5100c $\mathrm{c}^{\mathrm{TM}}$ (Covidien, Boulder, CO, USA), a trend monitor, FORE-SIGHT ${ }^{\mathrm{TM}}$ (CAS Medical Systems Inc, Branford, CT, USA), and NONIN EQUANOX $7600^{\mathrm{TM}}$ (Nonin Medical Inc, Plymouth, MN, USA) that both are FDA approved for absolute measurements.

\section{Materials and methods}

This study was approved by the local Ethics Committee (Journal no. H-3-2012-004) and conducted at the national university hospital, Rigshospitalet, Denmark. Written informed consent was obtained from the participants before inclusion. The study was registered at clinicaltrials.gov (ID NCT01552785).
Recruitment of healthy volunteers was accomplished by an ad in a local university paper. Inclusion criteria included normal health and a skinfold thickness of less than $10 \mathrm{~mm}$ on the lower arm measured by the Harpeden caliper to minimise the contribution of subcutaneous tissue. Exclusion criteria were pregnancy, hypertension, peripheral vascular disease, and local skin disease.

All measurements were done with subjects placed in upright position with the lower arm at heart level. The outline of each sensor was marked by a pen on the upper part of the flexor muscles of the lower arm to ensure that the sensors were applied to the same spot each time.

\subsection{Absolute values and repeatability}

The absolute $\mathrm{rStO}_{2}$ values of each device and repeatability of measurements during hemodynamic steady state were tested with one device at a time. First 20 seconds (s) of measurement was repeated 10 times at the marked site with total sensor lift up between repeats (Area 1 Fig. 1). As sensor repositioning in the clinical setting is likely to be at a slightly different site the sequence was repeated with the sensor being re-positioned at slightly differing sites around the area (Area 2 Fig. 1). This way an estimate of both the 'optimal' and the 'clinical' repeatability is achieved. This gave $2 \times 10$ steady state measurements with each device on each participant.

\subsection{Sensitivity to changes in oxygenation}

Secondly testing of device sensitivity to changing oxygenation was done with a vascular occlusion test inflating a cuff around the upper arm at heart level to more than $250 \mathrm{mmHg}$. $20 \mathrm{~s}$ of baseline $\mathrm{rStO}_{2}$ with each device was recorded before the cuff was inflated. $10 \mathrm{~s}$ after cuff inflation the different sensors were applied at the same site (Area 3 Fig. 1) sequentially to obtain three 1-min cycles of measurement of down-sloping $\mathrm{rStO}_{2}$. Each device measured $20 \mathrm{~s}$ within each cycle. After cuff deflation each device recorded $10 \mathrm{~s}$ of $\mathrm{rStO}_{2}$ during the short steady state of subsequent hyperperfusion (Fig. 2). The sequence of the

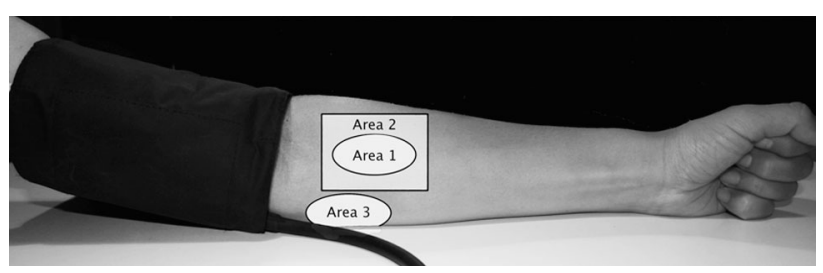

Fig. 1 The areas on the arm used for the measurements. Area 1 is the marked area for same site repeated measuresments, Area 2 is the area of repeated measurement at different sites, and Area 3 is the marked area of sensor position during cuff occlusion 


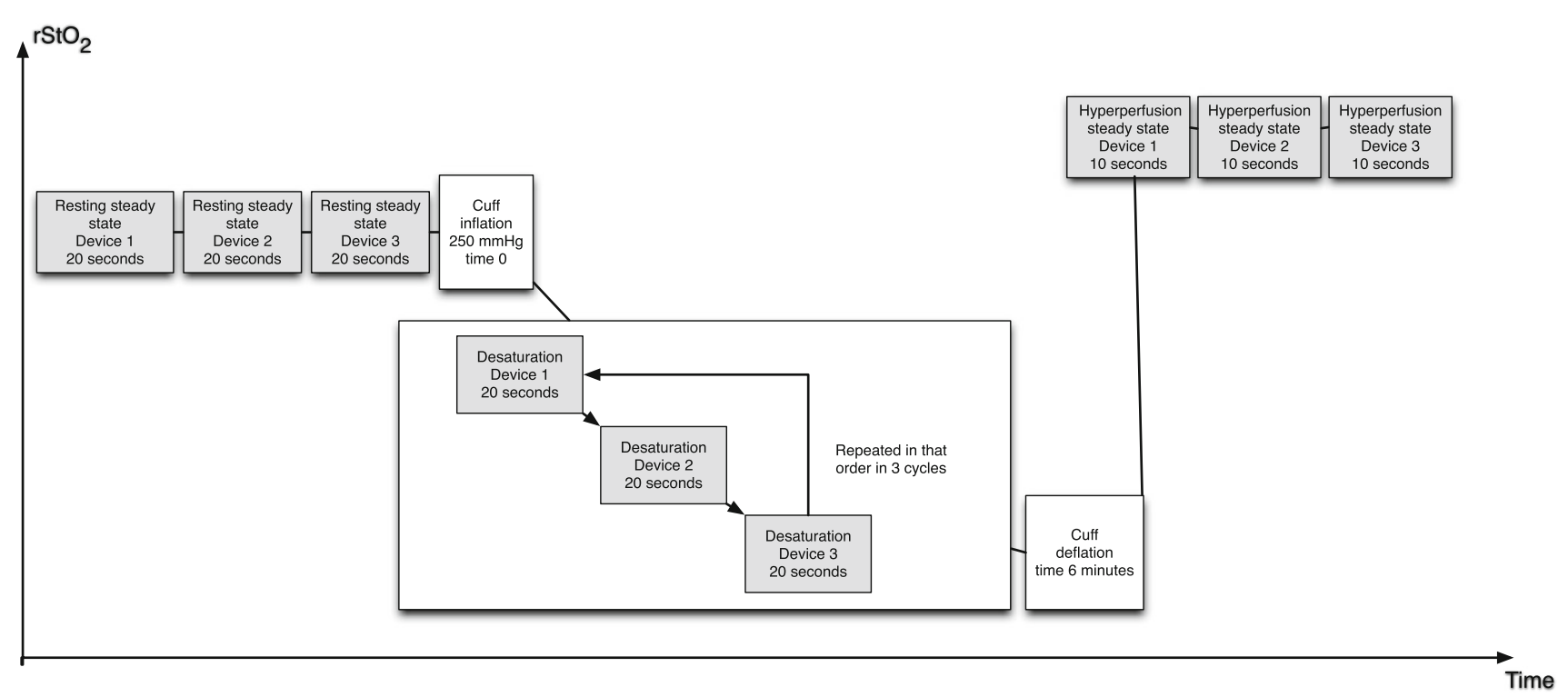

Fig. 2 Schematic presentation of the measurements during cuff inflation and deflation

three devices was changed between occlusions and six occlusions per subject were done ensuring a balanced design.

As the adhesive strength of the sensors wears off with repeated measurements the sensors were held in place by hand. This was found to be the most reliable way to get stable measurements, while great care was taken not to induce pressure on the tissue while avoiding possible source-to-detector light piping. All sensors were positioned so that the light path was parallel to the longitudinal direction of the muscles below. The experiment typically lasted 180 minutes in total per subject.

\subsection{NIRS instruments}

The three NIRS devices tested all use the multi-distance approach.

INVOS $5100 c^{\mathrm{TM}}$ with adult SomaSensor ${ }^{\mathrm{TM}}$ (SAFB-SM) uses two LED sources (730 and $810 \mathrm{~nm}$ ) at one position and two photodiode detectors at a distance of 3 and $4 \mathrm{~cm}$, and "subtracts" the short distance signal from the longer distance in order to diminish the contribution of the skin and scalp.

NONIN EQUANOX ${ }^{\mathrm{TM}} 7600$ with EQUANOX Advan$\mathrm{ce}^{\mathrm{TM}}$ Sensor, Model $8004 \mathrm{CA}^{\mathrm{TM}}$ uses four LEDs (730, 760, 810 , and $880 \mathrm{~nm}$ ) in each of two positions $6 \mathrm{~cm}$ apart with two photodiode detectors $2 \mathrm{~cm}$ apart in between in a symmetrical design.

FORE-SIGHT $^{\mathrm{TM}}$ Cerebral Oximeter with the medium sensor uses a 4-wavelength $(690,779,808$, and $850 \mathrm{~nm})$ laser source delivered to the sensor through an optical fibre and two photodiode detectors 1.25 and $4 \mathrm{~cm}$ from the source, respectively.
We will refer to the measured regional tissue oxygenation as $\mathrm{rStO}_{2}$ irrespective of instrument.

\subsection{Statistical analysis}

The a priori hypothesis was that the instruments have similar absolute values.

Sample size was calculated to have a power of $80 \%$ to detect a 5-percentage points difference between absolute values with a standard deviation of $5 \%$ and a paired design. A repeated measures general linear model tested differences in absolute values between devices with device as within-subject factor. Residual plots were visually assessed to assure reasonable fit. If data were either highly skewed or the variance was heterogeneous among groups a related samples Friedman test was used. If a significant device effect was found, post hoc pair-wise comparison was carried out.

Repeatability for each instrument was determined by one-way ANOVA with subject as the factor. The withinsubject standard deviation, $S_{w}$, was then estimated from the square root of the residual mean square. The $95 \% \mathrm{CI}$ being \pm 1.96 times $\mathrm{S}_{\mathrm{w}} / \operatorname{root}(2 \mathrm{n}(\mathrm{m}-1))$, where $\mathrm{n}$ is number of subjects and $\mathrm{m}$ the number of observations per subject.

To assess the dynamic properties of each device a simple, least square linear regression was applied to each $20 \mathrm{~s}$ sample of $\mathrm{rStO}_{2}$ recording during occlusion based on the approximation that the decline in $\mathrm{rStO}_{2}$ over time is linear during the first $3 \mathrm{~min}$ of desaturation [13, 14].

Two different approaches to estimate a signal-to-noise ratio (SNR) for each device were used. For each device on each subject a SNR was derived from the mean of the first cycle slopes divided by the same-site within-subject 
standard deviation (slope-SNR). Secondly data from the steady-state measurements were used. From a one-way ANOVA with the $20 \mathrm{~s}$ measurement as factor the spontaneous within-measurement fluctuations in $\mathrm{rStO}_{2}$ was used as signal while the between measurements variance was the noise term (variance-SNR). All data were log-transformed before the SNR analyses to achieve normal distribution.

Data were tested for normality with the Sharpiro Wilk test. Results from data with normal distribution are reported as mean with $95 \% \mathrm{CI}$, whereas all other results are given as median with interquartile range. A $p$ value below 0.05 was considered significant. Bonferroni adjustment was applied where appropriate.

All data was analysed in a Matlab versus R2011b (The MathWorks, Inc, Natick, MA, USA) script. All statistical tests were done in SPPS statistics version 20 (IBM, Corp, Armonk, NY, USA).

\section{Results}

10 subjects ( 5 male/5 female) healthy adults with an age range of 21-29 years and a median skinfold of $5.2 \mathrm{~mm}$ (IQR $3 \mathrm{~mm}$ ) were included.

\subsection{Absolute values}

During resting steady state the overall median $\mathrm{rStO}_{2}$ was $70.7 \%$ (IQR $7.7 \%$ ), $68.4 \%$ (IQR $8.4 \%$ ), and $64.6 \%$ (IQR 4.8) with the INVOS, NONIN, and FORE-SIGHT, respectively (Table 1; Fig. 3). In pairwise comparison only INVOS versus FORE-SIGHT were significantly different $(p=0.01)$.

During post-cuff-deflation reperfusion the median $\mathrm{rStO}_{2}$ was $85.3 \%$ (IQR $11.3 \%$ ) with INVOS, $88.0 \%$ (IQR $14.0 \%$ ) with NONIN, and $77.7 \%$ (IQR $10.5 \%$ ) with

Table 1 The absolute values of $\mathrm{rStO}_{2}$ of each device

\begin{tabular}{lllll}
\hline Device & Position & $\mathrm{N}$ & Median $(\%)$ & IQR $(\%)$ \\
\hline INVOS & Same site & 100 & 70.5 & 8.0 \\
& Various sites & 100 & 71.0 & 8.5 \\
& Total & 200 & 70.7 & 7.7 \\
NONIN & Same site & 100 & 67.0 & 8.3 \\
& Various sites & 100 & 69.5 & 8.6 \\
& Total & 200 & 68.4 & 8.4 \\
FORE-SIGHT & Same site & 100 & 63.8 & 4.7 \\
& Various sites & 100 & 65.8 & 6.3 \\
& Total & 200 & 64.6 & 4.8 \\
Total & Same site & 300 & 66.7 & 8.5 \\
& Various sites & 300 & 68.6 & 8.5 \\
& Total & 600 & 67.7 & 8.8 \\
\hline
\end{tabular}

FORE-SIGHT. The FORE-SIGHT was significantly lower than the two other devices $(p<0.0001)$ in both pair-wise comparisons.

\subsection{Repeatability}

The repeatability of FORE-SIGHT was best, while the repeatability of NONIN was worst (Table 2; Fig. 3). There was no difference between same-site repeatability and various-site repeatability.

\subsection{Sensitivity to changes in oxygenation}

The slopes of desaturation were steepest with NONIN $(p<0.0001)$ in both pair-wise comparisons, while the slopes of INVOS and FORE-SIGHT were similar (Table 3). Often both NONIN and INVOS reached their lower limit of recording before the start of third cycle of measurement giving an unequal number of observations per device, thus both analysis of data from all three cycles and only the first cycle was done giving the same main results (Fig. 4).

\subsection{Signal-to-noise ratio}

Both the cuff-induced slope-SNRs and spontaneous variance-SNRs were similar among devices. Slope-SNRs were $22.0 \mathrm{~min}^{-1}$ (CI 18.8-25.7), $23.8 \mathrm{~min}^{-1}$ (CI 19.7-28.8), and $22.0 \mathrm{~min}^{-1}$ (CI 17.8-27.1) with INVOS, NONIN, and FORE-SIGHT, respectively ( $p=0.5$ ). The variance-SNRs were 0.26 (CI 0.19-0.36), 0.24 (CI 0.18-0.32), and 0.21 (CI 0.16-0.26) with INVOS, NONIN, and FORE-SIGHT, respectively $(p=0.3)$.

\section{Discussion}

\subsection{Important findings}

The median resting steady state absolute values of the three oximeters were within 6 percentage points, whereas the values during deoxygenation were markedly different. The repeatability of FORE-SIGHT was best, while NONIN had the steepest slope of change during deoxygenation. The three devices had similar signal-to-noise ratios. Figure 3 illustrates this as the NONIN can be seen to have both the largest within-subject as well as between-subject variance.

\subsection{Appraisal of method}

This study was designed to compare three commercial NIRS oximeters and hypothesized that the devices give similar readings. The three sensors have quite different 
Fig. 3 The results from the steady-state same-site and various-site measurements. INVOS is red, NONIN green, and FORE-SIGHT blue (presented in that order from left to right). Each device-subject pair has the same-site measurements to the left and various-site measurements to the right. The boxes represents the interquartile ranges, the whisker the range, and the horizontal bar the median. Outliers are marked by a red plus symbol

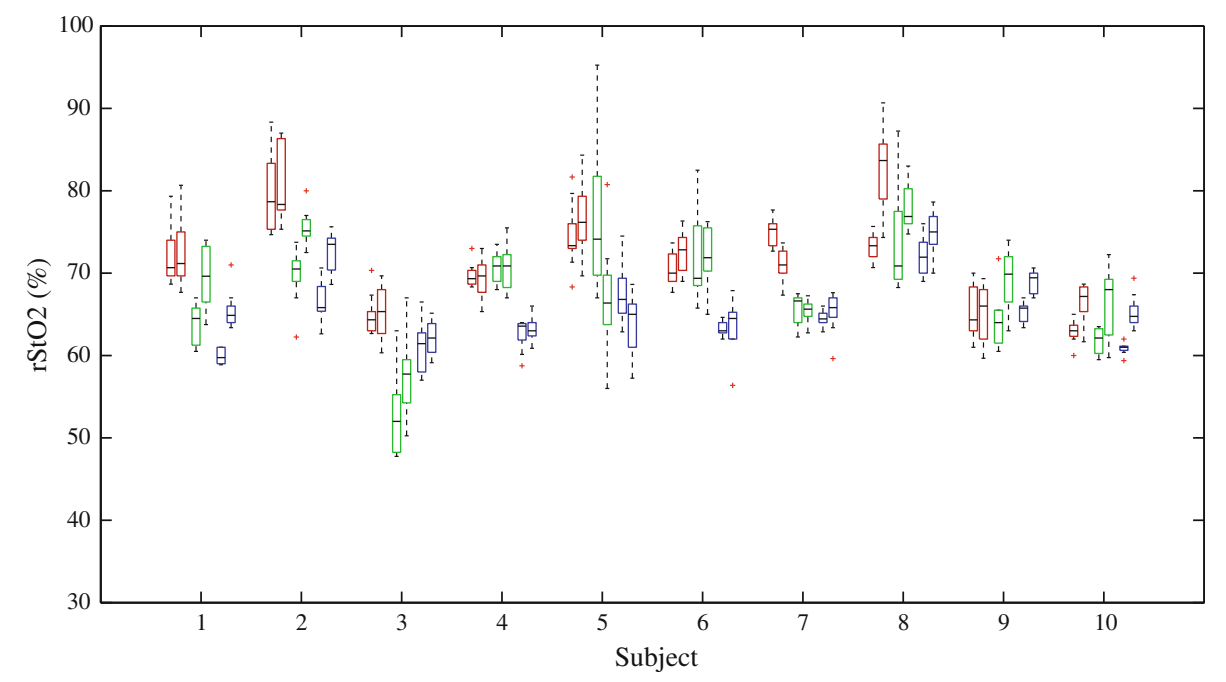

Table 2 The repeatability of same site and various site repositionings

\begin{tabular}{llllll}
\hline & \multicolumn{2}{l}{ Same site } & & \multicolumn{2}{c}{ Various sites } \\
\cline { 2 - 3 } \cline { 6 - 7 } & $\mathrm{S}_{\mathrm{w}}(\%)^{\mathrm{a}}$ & $\mathrm{CI}(\%)^{\mathrm{b}}$ & & $\mathrm{S}_{\mathrm{w}}(\%)^{\mathrm{a}}$ & $\mathrm{CI}(\%)^{\mathrm{b}}$ \\
\hline INVOS & 2.9 & $2.4-3.3$ & 3.6 & $3.0-4.1$ \\
NONIN & 4.6 & $3.9-5.3$ & 3.8 & $3.3-4.4$ \\
FORE-SIGHT & 2.0 & $1.7-2.3$ & 2.4 & $2.0-2.7$ \\
\hline
\end{tabular}

${ }^{\mathrm{a}} \mathrm{S}_{\mathrm{w}}$ : the within-subject standard deviation of the 10 repeated measurements

${ }^{\text {b }}$ CI: $95 \%$ confidence interval

Table 3 The dynamic ranges

\begin{tabular}{llclc}
\hline & $\begin{array}{l}\text { First cycle } \\
\text { slopes }(\% / \mathrm{min})\end{array}$ & $\begin{array}{l}\text { IQR } \\
(\% / \mathrm{min})\end{array}$ & $\begin{array}{l}\text { All slopes } \\
(\% / \mathrm{min})\end{array}$ & $\begin{array}{l}\text { IQR } \\
(\% / \mathrm{min})\end{array}$ \\
\hline INVOS & 13.2 & 9.6 & 10.4 & 8.5 \\
NONIN & 22.8 & 18.0 & 21.8 & 18.0 \\
FORE-SIGHT & 10.8 & 6.0 & 8.5 & 5.7 \\
\hline
\end{tabular}

light source and detector geometries and it could be argued that the volumes of tissue being examined are so different, that systematic difference are to be expected. However, if the overall goal is to provide a single, simple measure of tissue oxygenation for clinical use then similarity among oximeters is necessary.

The strength of our method is that it both gives an estimate of repeatability together with a measurement of the sensitivity to changing oxygenation. The method is easily reproducible and allows fast comparison of several instruments in healthy subjects with almost no risk. Vascular occlusion by cuff is a simple and robust way of inducing maximal tissue deoxygenation, allowing efficient estimation of device sensitivity. The response to cuff inflation and subsequent deflation probably varied during the six runs in each subject, but as our test design with each devices measuring on every round in a balanced manner, it is unlikely to have caused bias.

One important limitation of our study is that total occlusion of blood flow by a manually inflated cuff is not physiological. It results in a slight increase in blood volume due to the arterial inflow while the cuff pressure builds up and the venous outflow is already occluded. Thus the arterial to venous volume ratio $(\mathrm{A}: \mathrm{V}$ ratio) is likely to decrease. In systemic hypoperfusion and sepsis the $\mathrm{A}: \mathrm{V}$ ratio probably increases so the translation to clinical measurements may not be straightforward. Moreover the adhesive strength of the sensors wears off with repeated repositionings so the sensors were held by hand. Small differences in local pressure squeezing blood from superficial vessels cannot be completely excluded, but great care was taken and it is unlikely to have influenced the measurements much. Lastly it should be noted that these sensors are primarily intended for continuous monitoring whereas spot measurements are the cornerstone of this study. There are, however, no physiological or technical reason to believe that a reliable baseline measurement cannot be achieve within $10 \mathrm{~s}$ of stable readings as the used NIRS devices do not require calibration procedures or warm up.

\subsection{Comparison with previous studies}

Our results compare reasonably well with previous findings. The median baseline values measured in the range of 65-75\% were slightly higher with the INVOS. This is in accordance with other studies [15-17] While in our previous similar study INVOS 5100C with the same adult sensor had a similar mean $\mathrm{rStO}_{2}$ of $70.2 \pm 6.7 \%$ [5]

It is interesting that the $\mathrm{S}_{\mathrm{w}} \mathrm{s}$ of all instruments were quite low, meaning better repeatability than expected. Most 


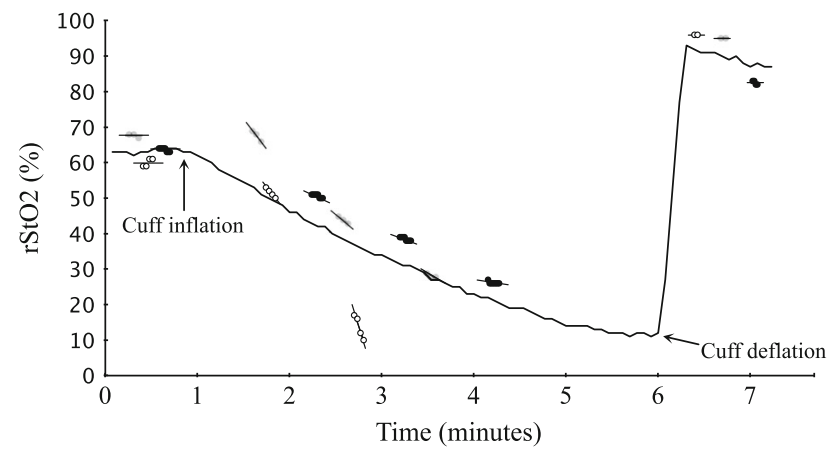

Fig. 4 An example of the first vascular occlusion test on a subject. The grey dots are INVOS; the black dots are FORE-SIGHT; the blank dots are NONIN. The continuous tracing is from another occlusion, but is included to illustrate the typical oxygenation trend. The horizontal lines represent steady state measurements. The regression lines are included for each of the $20 \mathrm{~s}$ measurements during the occlusion. Note that NONIN only have two measurements during the occlusion due to loss of signal

studies have reported repeatability of about $5-6 \%[3,4$, $18,19]$. In respect to the INVOS we have no good explanation for the difference in repeatability compared to our own, previous study, since both methods and subjects were similar.

To our knowledge these instruments haven't been tested and compared on muscle before. Data from the brain suggest that the FORE-SIGHT has better repeatability than INVOS [16]. Comparing the three instruments with a reference $\mathrm{rStO}_{2}$ calculated from jugular bulb and arterial blood in the human adult the accuracy root mean square was $4.26 \%$ with FORE-SIGHT, $9.69 \%$ with INVOS, and $6.27 \%$ with NONIN [15]. While the accuracy root mean square is not straightforwardly comparable to the $S_{w}$ in the present study the differences between instruments should be the same. It is thus again noticeable that INVOS performed better in our study.

The InSpectra Model 325 (Hutchinson Technology Inc., Hutchinson, MN, USA) is a NIRS device specifically designed for peripheral measurements only. Comparing the slopes of desaturations in \%/min of INVOS and FORESIGHT from present study with a study using the InSpectra on the forearm and thenar muscle shows reasonable agreement [20].

$\Delta \mathrm{rStO}_{2}$ in a recent study comparing the changes in $\mathrm{rStO}_{2}$ during a circumferential pneumatic head cuff inflation to estimate the contribution of extra-cranial scalp tissue were $16.6 \pm 9.6,11.8 \pm 5.3$, and $6.8 \pm 6.0 \%$ with INVOS, FORE-SIGHT, and NONIN, respectively [17]. Interestingly the ratio of $\Delta \mathrm{rStO}_{2 \text { (INVOS) }} / \Delta \mathrm{rStO}_{2 \text { (FORE-SIGHT) }}$ are similar in our study. This suggests that the differences in dynamics found on the arm could be similar on the head, and that the differences in so-called removal of extra-cranial tissue contamination as presented in the study by
Davie and Grocott could be simply a matter of different sensitivity to overall oxygenation changes in the tissue. The results with the NONIN are not comparable to present study as the classic Model $8000 \mathrm{CA}$ sensor was used, that previously has been found to be less sensitive than the new Model 8004CA [15], which was the sensor used in our study.

\subsection{Clinical relevance}

It is a key problem of NIRS tissue oximetry that there is no reference standard. We did not draw arterial and venous blood samples, as it is uncertain how well the venous saturation reflects the tissue oxygenation in a state of no flow. The fact that NONIN and INVOS often reached their lower boundary values while FORE-SIGHT showed continuing deoxygenation at a level of 20-30 \% suggests that NONIN and INVOS overestimate true deoxygenation in the adult human forearm. It is not certain that FORE-SIGHT is closer to the 'true' values, but at least the continued decline in $\mathrm{rStO}_{2}$ is likely to represent truly changing oxygenation.

It is noticeable that the results suggest that the variability of repeated measurements is independent of whether the sensors are repositioned at same site or at a different site. The implies at least on the lower arm that the exact sensor position is less important and that the source of variance is not so much differences in muscle oxygenation between adjacent tissues, but perhaps more a combination of physiologic fluctuations and optical heterogeneity on a smaller scale—such as hair and subcutaneous blood vessels in the light path.

When NIRS oximetry is used for trend monitoring the repeatability of measurement is less important, but good repeatability is paramount if NIRS is to be used as a spot measurement or if the monitoring is started when the patient status is uncertain, e.g. on admission to critical care. Moreover in critical care the sensor has to be repositioned during prolonged monitoring to avoid skin damage. The dynamic sensitivity in relation to repeatability is equally important since it is an estimate of the reliability of potential hypoxic levels of tissue oxygenation. If, for the sake of argument, it is assumed that a jugular bulb saturation below $50 \%$ indicates cerebral ischemia, then with a A: $\mathrm{V}$ ratio of 25:75 this theoretically corresponds to $\mathrm{rStO}_{2}$ $61 \%$, if the $\mathrm{SaO}_{2}$ is $95 \%$, thus with a $\mathrm{S}_{\mathrm{w}}$ of $5 \%$ a spot measurement should be below $(61 \%-1.96 \times 5 \%)$ $52 \%$ to be more than $95 \%$ certain that the tissue is actually ischemic and above $(61 \%+1.96 \times 5 \%) 71 \%$ to be certain that it is not. If in addition the sensitivity to changes is low the risk of undetected true hypoxia will be high. The actual changes in oxygenation induced by cuff inflation-deflation, is unknown in the current setup, but it is safe to say that the three tested devices do not agree about 
the magnitude of changes. A pragmatic approach would be to care less about the closeness to the 'true' values, but to settle for the device with the best combination of repeatability and sensitivity to changing oxygenation. However our results suggest that high sensitivity comes at the expense of good repeatability, as our estimates of signal-tonoise ratios for each device were similar.

The lack of a reference standard is a drawback for the technology and it would be a large step forward if an international standard could be determined. One problem with in vivo testing as done in the present study is that results may or may not be generalizable to other tissues. It is thus important to test and compare the commercial available NIRS equipment on different sites in different populations. In-vitro phantom testing could also prove useful, but has not been widely used with commercial equipment, even though it could perhaps provide a possible framework for a future standardization procedure.

\section{Conclusion}

In conclusion we found that INVOS $5100 \mathrm{C}$ with the adult SomaSensor, NONIN EQUANOX 7600 with sensor 8004CA, and FORE-SIGHT with medium sensor all had pair-wise differences in either median absolute values, repeatability, and/or dynamic measurements. A standardized testing procedure would be a step forward for the technology.

Acknowledgments This project was funded by a grant from the Elsass Foundation.

Conflict of interest The authors declare that they have no conflict of interest.

Open Access This article is distributed under the terms of the Creative Commons Attribution License which permits any use, distribution, and reproduction in any medium, provided the original author(s) and the source are credited.

\section{References}

1. Naulaers G, Meyns B, Miserez M, Leunens V, van Huffel S, Casaer $\mathrm{P}$, et al. Use of tissue oxygenation index and fractional tissue oxygen extraction as non-invasive parameters for cerebral oxygenation. A validation study in piglets. Neonatology. 2007;92:120-6.

2. Fenik JC, Rais-Bahrami K. Neonatal cerebral oximetry monitoring during ECMO cannulation. J Perinatol. 2009;29:376-81.

3. Sorensen LC, Greisen G. Precision of measurement of cerebral tissue oxygenation index using near-infrared spectroscopy in preterm neonates. J Biomed Opt. 2006;11:054005.
4. Pocivalnik M, Pichler G, Zotter H, Tax N, Muller W, Urlesberger B. Regional tissue oxygen saturation: comparability and reproducibility of different devices. J Biomed Opt. 2011;16:057004.

5. Hyttel-Sørensen S, Sorensen LC, Riera J, Greisen G. Tissue oximetry: a comparison of mean values of regional tissue saturation, reproducibility and dynamic range of four NIRS-instruments on the human forearm. Biomed Opt Express. 2011;2:3047-57.

6. Rolfe P. In vivo near-infrared spectroscopy. Annu Rev Biomed Eng. 2000;2:715-54.

7. Payen D, Luengo C, Heyer L, Resche-Rigon M, Kerever S, Damoisel $\mathrm{C}$, et al. Is thenar tissue hemoglobin oxygen saturation in septic shock related to macrohemodynamic variables and outcome? Crit Care. 2009;13(Suppl 5):S6.

8. McKinley BA, Marvin RG, Cocanour CS, Moore FA. Tissue hemoglobin $\mathrm{O}_{2}$ saturation during resuscitation of traumatic shock monitored using near infrared spectrometry. J Trauma. 2000;48:637-42.

9. Pareznik R, Knezevic R, Voga G, Podbregar M. Changes in muscle tissue oxygenation during stagnant ischemia in septic patients. Intensive Care Med. 2006;32:87-92.

10. Lima A, van Bommel J, Jansen TC, Ince C, Bakker J. Low tissue oxygen saturation at the end of early goal-directed therapy is associated with worse outcome in critically ill patients. Crit Care. 2009;13(Suppl 5):S13.

11. Scheeren TWL, Schober P, Schwarte LA. Monitoring tissue oxygenation by near infrared spectroscopy (NIRS): background and current applications. J Clin Monit Comput. 2012;26:279-87.

12. MacLeod D, Ikeda K, Morretti E, Keifer J, Ames W. Validation of the CAS adult cerebral oximeter during hypoxia in healthy volunteers. Anesth Analg. 2006;102:S162.

13. Ferrari M, Muthalib M, Quaresima V. The use of near-infrared spectroscopy in understanding skeletal muscle physiology: recent developments. Philos Trans A Math Phys Eng Sci. 2011;369:4577-90.

14. Felici F, Quaresima V, Fattorini L, Sbriccoli P, Filligoi GC, Ferrari M. Biceps brachii myoelectric and oxygenation changes during static and sinusoidal isometric exercises. J Electromyogr Kinesiol. 2009;19:e1-11.

15. Bickler PE, Feiner JR, Eilers H, Rollins M. Performance of 5 cerebral oximeters during hypoxia in healthy volunteers. American Society of Anesthesiologists Annual meeting. 2011. pp. 1-2.

16. MacLeod D, Ikeda K, Vacchiano C. Simultaneous comparison of FORE-SIGHT and INVOS cerebral oximeters to Jugular Bulb and arterial co-oximetry measurements in healthy volunteers. Anesth Analg. 2009;108:1-104.

17. Davie SN, Grocott HP. Impact of extracranial contamination on regional cerebral oxygen saturation: a comparison of three cerebral oximetry technologies. Anesthesiology. 2012;116:834-40.

18. Wijbenga RG, Lemmers PMA, van Bel F. Cerebral oxygenation during the first days of life in preterm and term neonates: differences between different brain regions. Pediatr Res. 2011;70:389-94.

19. Dullenkopf A, Kolarova A, Schulz G, Frey B, Baenziger O, Weiss M. Reproducibility of cerebral oxygenation measurement in neonates and infants in the clinical setting using the NIRO 300 oximeter. Pediatr Crit Care Med. 2005;6:344-7.

20. Bezemer R, Lima A, Myers D, Klijn E, Heger M, Goedhart PT, et al. Assessment of tissue oxygen saturation during a vascular occlusion test using near-infrared spectroscopy: the role of probe spacing and measurement site studied in healthy volunteers. Crit Care. 2009;13(Suppl 5):S4. 